\title{
Study of heavy exotic baryon resonances
}

\author{
Yubing Dong ${ }^{1,2, \star, \star \star}$ and Qi-Fang Lü ${ }^{1, \star \star \star}$ \\ ${ }^{1}$ Institute of High Energy Physics, Chinese Academy of Sciences, Beijing 100049, P. R. China \\ ${ }^{2}$ Theoretical Physics Center for Science Facilities (TPCSF), CAS, Beijing 100049, P. R. China
}

\begin{abstract}
The possible production of the neutral $P_{c}(4380)$ and $P_{c}(4450)$ in the J-PARC experiment is discussed. Different spin-parity assignments of the $P_{c}$ states are considered and differential cross sections of $\pi^{-} p \rightarrow J / \psi p$ are calculated. Moreover, the strong decays of $P_{c} \rightarrow J / \psi p$ are estimated based on a $\Sigma_{c} \bar{D}^{*}$ molecular scenario. Our results disfavor all the $P-$ wave $\Sigma_{c} \bar{D}^{*}$ assignments, while favor the $S-$ wave $\Sigma_{c} \bar{D}^{*}$ picture of the two $P_{c}$ states comparing to the experimental data. In addition, we also analyze the possible $J^{P}=3 / 2^{-}$state in $\Sigma_{c}^{*} \bar{D}$ and $\Sigma_{c}^{*} \bar{D}^{*}$ pictures with heavy quark limit.
\end{abstract}

\section{Introduction}

It is well-known that two hidden charm pentaquark resonances $P_{c}^{+}(4380)$ and $P_{c}^{+}(4450)$ were reported by the LHCb Collaboration in the process of $\Lambda_{b}^{0} \rightarrow J / \psi K^{-} p$ [1]. Their masses and widths are $(4380 \pm 8 \pm 29 ; 205 \pm 18 \pm 86) \mathrm{MeV}$ for $P_{c}(4380)$ and $(4449.8 \pm 1.7 \pm 2.5 ; 39 \pm 5 \pm 19) \mathrm{MeV}$ for $P_{c}(4450)$, respectively. Their isospins are $I=1 / 2$. Till now, the spin-parities of the two states are not clear, and some possible spin-parity assignments of $\left(3 / 2^{-}, 5 / 2^{+}\right),\left(3 / 2^{+}, 5 / 2^{-}\right)$, and $\left(5 / 2^{+}, 3 / 2^{-}\right)$are suggested by the experimental analyses. Due to their strong decay mode of $J / \psi p$, the two states are expected to have five quark content $u u d c \bar{c}$. The branching fractions $\mathcal{B}\left(\Lambda_{b}^{0} \rightarrow P_{c}^{+} K^{-}\right) \mathcal{B}\left(P_{c}^{+} \rightarrow J / \psi p\right)$ of the decays $\Lambda_{b}^{0} \rightarrow J / \psi K^{-} p$ are given by [2]. In addition, the two $P_{c}$ states were also confirmed in the process of $\Lambda_{b}^{0} \rightarrow J / \psi \pi^{-} p$ [3].

Since the observation of $X(3872)$, many exotic mesons $X Y Z$ particles have been reported. Those states cannot be easily understood as the $q \bar{q}$ mesons due to their narrow widths and their masses very near to the thresholds of the two mesons $[4,5]$. Most of them, particularly the charged $Z_{c}$ and $Z_{b}$, should have four-quark content. The newly observation of the two charged $P_{c}$ states provides a unique perspective to the exotic baryon states. There are many interesting studies on these two $P_{c}$ states in the literature. They may be interpreted as the compact pentaquark states, where the five quarks are tightly bounded and interact with each other via color magnetic interaction directly [6, 7]. They are also regarded as the loosely bound molecular states composed of one meson and one baryon [8-13]. Moreover, the kinematical effects due to the re-scattering processes on the two states have been carefully discussed $[14,15]$. It should be stressed that besides their mass spectrum, their

\footnotetext{
${ }^{\star}$ This work is supported by National Sciences Foundations of China No. 11475192 as well as supported, in part, by the DFG and the NSFC through funds (11621131001) provided to the Sino-German CRC 110 "Symmetries and the Emergence of Structure in QCD”.

$\star \star$ e-mail: dongyb@ihep.ac.cn

$\star \star \star$ e-mail: lvqifang@ihep.ac.cn
} 
production in other facilities and the decay behaviors can also provide information about their intrinsic structures. [12] has discussed the $P_{c}(4450) \rightarrow J / \psi p$ partial decay width, by regarding it as a bound state of charmonium $\psi(2 S)$ and nucleon, and [16] performed a comprehensive calculation based on the heavy quark symmetry and spin rearrangement scheme, where the $\Sigma_{c}^{*} \bar{D}, \Sigma_{c} \bar{D}^{*}$, and $\Sigma_{c}^{*} \bar{D}^{*}$ molecular candidates are all taken into account simultaneously. In the $\Lambda_{b}^{0} \rightarrow J / \psi K^{-} p$ decay process, only charged $P_{c}$ states can be observed. The $\gamma p \rightarrow J / \psi p$ reaction with charged $P_{c}$ production has been proposed for the future JLab. experiment [17-20]. In addition, the production of their neutral partners is expected to be observed in J-PARC experiment by our previous study [21].

This paper is organized as follows. In Sect. 2 we show the production of the neutral $P_{c}$ states in the future J-PARC experiment. In Sect. 3, the strong decay widths $J / \psi p$ under a $\Sigma_{c} \bar{D}^{*}$ molecule assignment with spin-parity $J^{P}=3 / 2^{ \pm}$or $5 / 2^{ \pm}$are estimated. In our calculation, we use a phenomenological effective Lagrangian approach. It should be noticed that this technique, for evaluating composite hadron systems, has been widely applied for various molecular states, where the compositeness condition corresponding to $Z=0$ has been employed [22-24]. Finally, a short summary is given in Sec. 4.

\section{Production of the two $P_{c}$ states at J-PARC}

We first study the $\pi^{-} p \rightarrow J / \psi n$ reaction within an effective Lagrangian approach [21]. The relevant Feynman diagrams are displayed in figure 1. The $s$-channel contributions from the $P_{c}$ states with different spin-parity assumptions are taken into account. The $u$-channel contribution is negligible due to the highly off-shell intermediate $P_{c}$ states. The background contributions from $t$-channel $\pi$ and $\rho$ meson exchanges are considered as well.
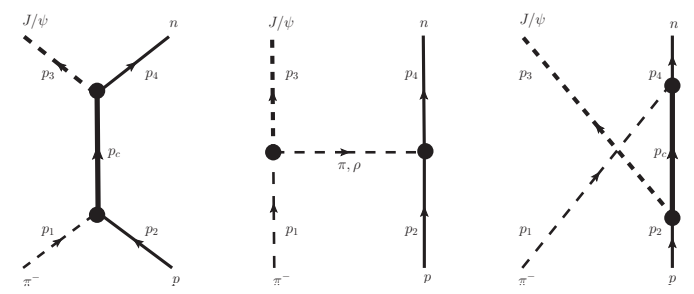

Figure 1. Feynman diagrams for $\pi^{-} p \rightarrow J / \psi n$ reaction.

The effective Lagrangians for $P_{c} N J / \psi$ couplings can be written as [25]:

$$
\mathcal{L}_{P_{c} N J / \psi}^{3 / 2^{ \pm}}=-\frac{i g_{1}}{2 M_{N}} \bar{N} \Gamma_{v}^{( \pm)} \psi^{\mu v} P_{c \mu}+\text { H.c., } \quad \mathcal{L}_{\mathrm{P}_{\mathrm{c}} \mathrm{NJ} / \psi}^{5 / 2^{ \pm}}=\frac{\mathrm{g}_{1}}{\left(2 \mathrm{M}_{\mathrm{N}}\right)^{2}} \overline{\mathrm{N}} \Gamma_{v}^{(\mp)} \partial^{\alpha} \psi^{\mu \nu} \mathrm{P}_{\mathrm{c} \mu \alpha}+\text { H.c. },
$$

for $J^{p}=3 / 2^{ \pm}$, and $5 / 2^{ \pm}$, respectively. In (1) the $\Gamma$ matrices are defined as $\Gamma_{\mu}^{+} \equiv \gamma_{\mu} \gamma_{5}$ and $\Gamma_{\mu}^{-} \equiv$ $\gamma_{\mu}$, respectively. In our calculation, the higher partial wave terms are neglected due to the small momentum of the final $J / \psi N$ states compared with nucleon mass and the lack of the experimental data [17]. The effective Lagrangians for $P_{c} N \pi$ vertexes are

$$
\begin{aligned}
\mathcal{L}_{P_{c} N \pi}^{3 / 2^{+}} & =\frac{g_{P_{c} N \pi}}{m_{\pi}} \bar{N} \vec{\tau} \cdot \partial_{\mu} \vec{\pi} P_{c}^{\mu}+\text { H.c., } \quad \mathcal{L}_{\mathrm{P}_{\mathrm{c}} \mathrm{N} \pi}^{3 / 2^{-}}=\frac{\mathrm{g}_{\mathrm{P}_{\mathrm{c}} \mathrm{N} \pi}}{\mathrm{m}_{\pi}^{2}} \overline{\mathrm{N}} \gamma_{5} \gamma_{\mu} \tilde{\tau} \cdot \partial^{\mu} \partial_{\nu} \tilde{\pi} \mathrm{P}_{\mathrm{c}}^{v}+\text { H.c. }, \\
\mathcal{L}_{P_{c} N \pi}^{5 / 2^{+}} & =\frac{g_{P_{c} N \pi}}{m_{\pi}^{3}} \bar{N} \gamma_{5} \gamma_{\mu} \vec{\tau} \cdot \partial^{\mu} \partial_{\nu} \partial_{\lambda} \vec{\pi} P_{c}^{v \lambda}+\text { H.c., } \quad \mathcal{L}_{\mathrm{P}_{\mathrm{c}} \mathrm{N} \pi}^{5 / 2^{-}}=\frac{\mathrm{g}_{\mathrm{P}_{\mathrm{c}} \mathrm{N} \pi}}{\mathrm{m}_{\pi}^{2}} \overline{\mathrm{N}} \tilde{\tau} \cdot \partial_{\mu} \partial_{v} \tilde{\pi} \mathrm{P}_{\mathrm{c}}^{\mu \nu}+\text { H.c. },
\end{aligned}
$$


Table 1. Coupling constants of $P_{c} N J / \psi$ and $P_{c} N \pi$ with different $J^{P}$ assignments by assuming the branching ratios are $10 \%$ and $1 \%$, respectively.

\begin{tabular}{||l|c|c|c|c|c||}
\hline \hline State & Channel & $3 / 2^{+}$ & $3 / 2^{-}$ & $5 / 2^{+}$ & $5 / 2^{-}$ \\
\hline$P_{c}(4380)$ & $J / \psi N$ & 1.09 & 0.49 & 2.17 & 5.13 \\
& $\pi N$ & $8.56 \times 10^{-3}$ & $3.43 \times 10^{-4}$ & $3.59 \times 10^{-5}$ & $8.95 \times 10^{-4}$ \\
& $J / \psi N$ & 0.41 & 0.20 & 0.80 & 1.75 \\
$P_{c}(4450)$ & $\pi N$ & $3.65 \times 10^{-3}$ & $1.43 \times 10^{-4}$ & $1.47 \times 10^{-5}$ & $3.75 \times 10^{-4}$ \\
& $\pi N$ \\
\hline \hline
\end{tabular}

for $J^{P}=3 / 2^{ \pm}$and $J^{p}=5 / 2^{ \pm}$, respectively. Furthermore, the couplings for $t$-channel diagram are also needed. We adopt the commonly used Lagrangian densities for $J / \psi \pi \pi, J / \psi \pi \rho, \pi N N$, and $\rho N N$ vertexes given by $[26,27]$.

The $P_{c} \rightarrow N J / \psi$ and $P_{c} \rightarrow N \pi$ decay processes are calculated and the relevant coupling constants can be obtained from the partial decay widths with different $J^{P}$ assignments. Assumed that the branching ratios are $10 \%$ and $1 \%$ for $P_{c} \rightarrow N J / \psi$ and $P_{c} \rightarrow N \pi$, respectively, the obtained coupling constants are listed in table 1 . To account for the fact that hadrons are not point-like particles, we also employ the form factors [28] in our calculations. For example, we have $F\left(q^{2}\right)=\frac{\Lambda_{P_{c}}^{4}}{\Lambda_{P_{c}}^{4}+\left(q^{2}-M_{P_{c}}^{2}\right)^{2}}$ for the intermediate $P_{c}$ in the S-channel diagram with the cutoff parameter $\Lambda_{P_{c}}=0.5 \mathrm{GeV}$ [25].

Then, the differential cross section is

$$
\frac{d \sigma}{d \cos \theta}=\frac{M_{N}^{2}}{16 \pi s} \frac{\left|\vec{p}_{3}^{c . m \cdot}\right|}{\left|\vec{p}_{1}^{c . m \cdot}\right|}\left|\mathcal{M}_{\pi^{-} p \rightarrow J / \psi n}\right|,
$$

in the center-of-mass (c.m.) frame with $\theta$ being the scattering angle of the outgoing $J / \psi$ relative to the incoming pion beam, and $\vec{p}_{1}^{c . m}$. and $\vec{p}_{3}^{\text {c.m. }}$ the three-momenta of $\pi$ and $J / \psi$ mesons in c.m. frame. Since the relative phase between different amplitudes is unknown, the interference terms between them are ignored here. This scheme avoids adding new free parameters and our calculation shows that the magnitudes of different contributions are much different, and hence the effects are expected to be small enough.

From our calculated total cross sections, we find that the $\pi$ and $\rho$ meson exchanges provide significant background contributions, while there are two narrow bump structures from $P_{c}^{0}(4380)$ and $P_{c}^{0}(4450)$ contributions. With the different $J^{P}$ assignments, the divergences among the total cross sections are not clear, which can hardly be used to identify the spin-parity of the two $P_{c}$ states. The peaks are in the magnitude of $1 \mu \mathrm{b}$ at the c.m. energy $\mathrm{W}=4.38 \mathrm{GeV}$ and $4.45 \mathrm{GeV}$, which can be measured in the future J-PARC experiment. It should be stressed that the two $P_{c}$ states are firstly observed in the $J / \psi p$ invariant mass, and this decay mode can occur via fallen apart mechanism. For the $\pi N$ channel, although the decay width is suppressed by $c \bar{c}$ pair annihilation, this process is still an OZI-allowed two-body strong decay with large phase space. Here, we assume the branching ratios for the two $P_{c}$ states to $J / \psi N$ and $\pi N$ channels being $10 \%$ and $1 \%$, respectively.

The estimated differential cross sections are shown in figure 2. It is seen that the $t$-channel meson exchanges provide forward contribution in the whole energy region. The differential cross sections at $4.38 \mathrm{GeV}$ and $4.45 \mathrm{GeV}$ are mainly from $P_{c}^{0}(4380)$ and $P_{c}^{0}(4450)$ contributions, respectively. The angular distributions of the two $P_{c}$ states are obviously different with the forward background contribution and display significant different behaviors with different $J^{P}$ assignments. These specific features may be observed by the future experiment at J-PARC and can help us to distinguish different spin-parity assumptions. 

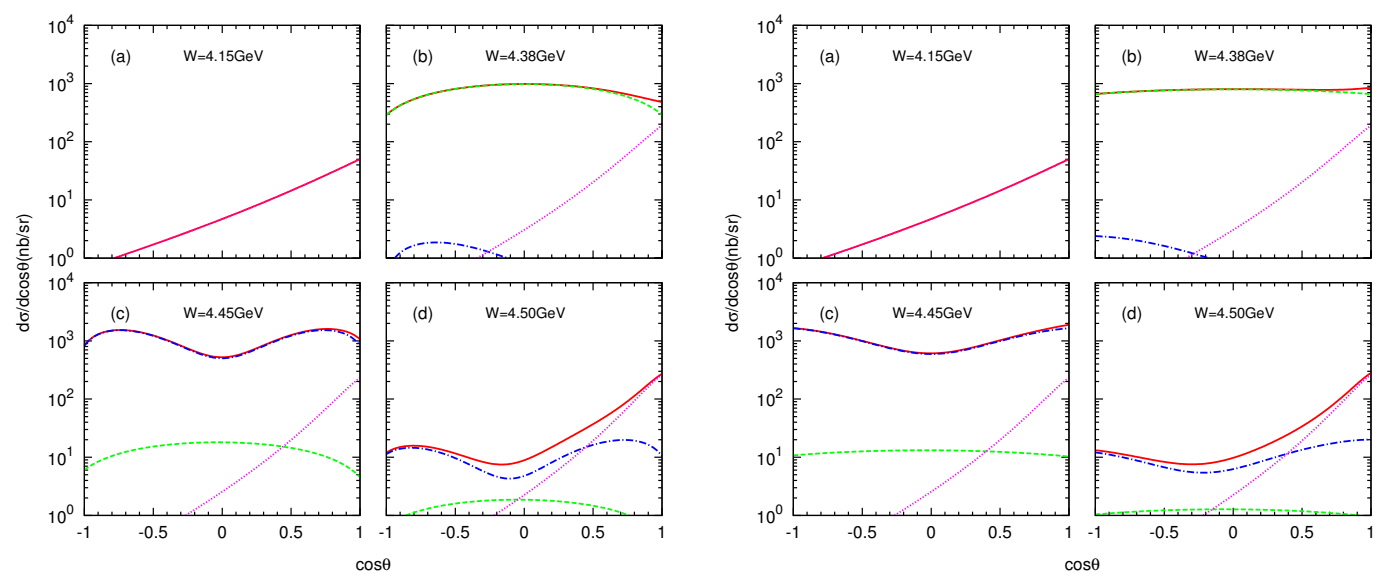

Figure 2. The differential cross sections for the $\pi^{-} p \rightarrow J / \psi n$ reaction at the c.m. energies $\mathrm{W}=4.15 \mathrm{GeV}, 4.38$ $\mathrm{GeV}, 4.45 \mathrm{GeV}$, and $4.45 \mathrm{GeV}$, respectively. The $\left(P_{c}^{0}(4380), P_{c}^{0}(4450)\right)$ respectively correspond to $\left(3 / 2^{+}, 5 / 2^{-}\right)$ (left panel) and $\left(3 / 2^{-}, 5 / 2^{+}\right)$(right panel) assumptions. The red solid, green dashed, blue dot-dashed, and pink short dotted curves stand for the total, $\left(P_{c}^{0}(4380)\right.$ and $\left.P_{c}^{0}(4450)\right)$, and the background contributions, respectively.

\section{Strong decays of the two $P_{c}$ states in a $\Sigma_{c} \bar{D}^{*}$ molecular scenario}

In this section, we show a calculation for the strong decays of the two $P_{c}$ states based on a $\Sigma_{c} \bar{D}^{*}$ molecule scenario [13]. The simplest effective Lagrangian describing the $P_{c} \Sigma_{c} \bar{D}^{*}$ couplings can be expressed as

$$
\begin{aligned}
& \mathcal{L}_{P_{c} \Sigma_{c} \bar{D}^{*}}^{3 / 2^{ \pm}}(x)=-i g_{P_{c} \Sigma_{c} \bar{D}^{*}} \int d^{4} y \Phi\left(y^{2}\right) \bar{\Sigma}_{c}\left(x+\omega_{\bar{D}^{*}} y\right) \Gamma^{( \pm)} \bar{D}_{\mu}^{*}\left(x-\omega_{\Sigma_{c}} y\right) P_{c}^{\mu}(x)+\text { H.c. } \\
& \mathcal{L}_{P_{c} \Sigma_{c} \bar{D}^{*}}^{5 / 2^{ \pm}}(x)=g_{P_{c} \Sigma_{c} \bar{D}^{*}} \int d^{4} y \Phi\left(y^{2}\right) \bar{\Sigma}_{c}\left(x+\omega_{\bar{D}^{*}} y\right) \Gamma^{(\mp)} \partial_{\mu} \bar{D}_{v}^{*}\left(x-\omega_{\Sigma_{c}} y\right) P_{c}^{\mu v}(x)+\text { H.c. }
\end{aligned}
$$

where $\omega_{\bar{D}^{*}}=m_{\bar{D}^{*}} /\left(m_{\Sigma_{c}}+m_{\bar{D}^{*}}\right)$ and $\omega_{\Sigma_{c}}=m_{\Sigma_{c}} /\left(m_{\Sigma_{c}}+m_{\bar{D}^{*}}\right)$. The $\Gamma^{( \pm)}$matrices are defined as $\Gamma^{+}=\gamma_{5}$ and $\Gamma^{-}=I$. The correlation function $\Phi\left(y^{2}\right)$ is chosen to describe the distribution of $\Sigma_{c}$ and $\bar{D}^{*}$ in $P_{c}$ states. In order to render the Feynman diagrams ultraviolet finite, its Fourier transform of $\Phi\left(y^{2}\right)$ should vanish fast in the ultraviolet region of the Euclidean space. Here, we adopt the commonly used Gaussian-type and thus, the Fourier transform can be written as $\tilde{\Phi}\left(p_{E}^{2} / \Lambda^{2}\right) \doteq \exp \left(-p_{E}^{2} / \Lambda^{2}\right)$, with $p_{E}$ being the Euclidean Jacobi momentum and $\Lambda$ a free size parameter characterizing the distribution of the two components in the molecule. This parameter is about $1 \mathrm{GeV}$ and may vary in different systems. The coupling constant $g_{P_{c} \Sigma_{c} \bar{D}^{*}}$ can be determined by the compositeness condition [29]. This condition requires that the renormalization constant of the hadronic molecular wave function is equal to zero, $Z_{P_{c}}=1-\partial \Sigma_{P_{c}}^{T( \pm)}(p) /\left.\partial \not p\right|_{\not p=m_{P_{c}}} \equiv 0$, where the $\Sigma_{P_{c}}^{T( \pm)}(p)$ is the transverse part of the mass operator. Then, the hadronic decay $P_{c}^{+} \rightarrow J / \psi p$, shown in figure 3 with the exchanging of $D^{*}, D$, and $\Sigma_{c}$ hadrons, can be estimated if the effective Lagrangians $\mathcal{L}_{\psi D^{(*)} D^{(*)}}$ and $\mathcal{L}_{\Sigma_{c} N D^{*}(D)}$ are known. Paper [13] gives their explicit expressions for the Lagrangians and the relevant coupling constants.

The calculated partial decay widths of $P_{c}^{+} \rightarrow J / \psi p$ are presented in table 2 with $\Lambda=1 \mathrm{GeV}$. In our calculation, we found the partial decay widths increase for $J^{P}=3 / 2^{ \pm}$assignment, while decrease for $J^{P}=5 / 2^{ \pm}$when $\Lambda$ increases. For the $P_{c}(4380)$ state, the predicted partial decay widths in all the 


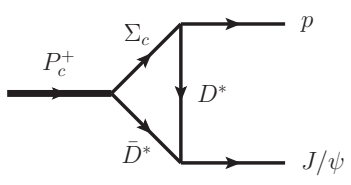

(a)

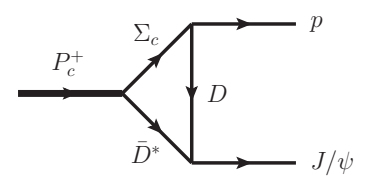

(b)

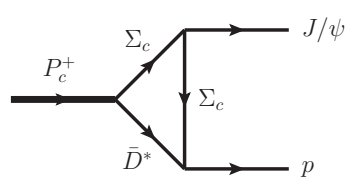

(c)

Figure 3. Feynman diagrams for the $P_{c}^{+} \rightarrow J / \psi p$ decay process.

four cases are all smaller than the experimental total width within the errors. Since the $J^{P}=5 / 2^{-}$ assignment is not favored by the experiment [1], and the $\Sigma_{c} \bar{D}^{*}$ molecule should be in $D$ wave in this case, the $J^{P}=5 / 2^{-}$assumption for $P_{c}(4380)$ is ruled out, while others are consistent with the present data. For the $P_{c}(4450)$ state, since $J^{P}=3 / 2^{+}$assignment is not favored by the LHCb collaboration [1] and the calculated partial decay width is much larger than the experimental total width, this case can be completely excluded as well. Also, the $J^{P}=5 / 2^{+}$case is disfavored due to the large predicted width, and the $D$ wave $\Sigma_{c} \bar{D}^{*}$ molecule with $J^{P}=5 / 2^{-}$can be hardly formed through the one boson exchange interaction. Hence, only the $S$ wave $\Sigma_{c} \bar{D}^{*}$ molecule is left for the $P_{c}(4450)$ state.

Table 2. Partial decay widths of $P_{c}^{+} \rightarrow J / \psi p$ with different $J^{P}$ assignments with $\Lambda=1 \mathrm{GeV}$. The unit is in MeV.

\begin{tabular}{|c|c|c|c|c||c|}
\hline State & $3 / 2^{+}$ & $3 / 2^{-}$ & $5 / 2^{+}$ & $5 / 2^{-}$ & Expt. [1] \\
\hline \hline$P_{c}(4380)$ & 173.12 & 38.12 & 169.51 & 4.96 & $205 \pm 18 \pm 86$ \\
\hline$P_{c}(4450)$ & 369.82 & 25.00 & 76.15 & 3.39 & $39 \pm 5 \pm 19$ \\
\hline \hline
\end{tabular}

Besides the $\Sigma_{c} \bar{D}^{*}$ molecular picture, the $\Sigma_{c}^{*} \bar{D}$ and $\Sigma_{c}^{*} \bar{D}^{*}$ scenarios for the two $P_{c}$ states are also proposed in the literature. In the heavy quark limit, the $S$-wave $D$ and $D^{*}$ mesons can be categorized into a doublet as well as the heavy baryons $\Sigma_{c}$ and $\Sigma_{c}^{*}$. Based on the symmetry and spin rearrangement scheme, [16] gave the ratios of the partial decay widths in the different molecular scenarios. This model independent result shows that for the three $S$-wave $\Sigma_{c} \bar{D}^{*}, \Sigma_{c}^{*} \bar{D}$, and $\Sigma_{c}^{*} \bar{D}^{*}$ molecules with $J^{P}=$ $3 / 2^{-}$, the ratios of their $J / \psi p$ decay widths satisfy the relation of $\Gamma\left[\left(\Sigma_{c} \bar{D}^{*}\right)\right]: \Gamma\left[\left(\Sigma_{c}^{*} \bar{D}\right)\right]: \Gamma\left[\left(\Sigma_{c}^{*} \bar{D}^{*}\right)\right]=$ $1.0: 2.7: 5.4$. Simply employing those ratios and the results shown in table 2 with $\Lambda=1 \mathrm{GeV}$, we can estimate the $J / \psi p$ decay widths of the two $P_{c}$ states in the $\Sigma_{c}^{*} \bar{D}$ and $\Sigma_{c}^{*} \bar{D}^{*}$ molecular scenarios with $J^{P}=3 / 2^{-}$. The obtained results of $\Gamma_{P_{c}(4380)}^{3 / 2-}\left[\left(\Sigma_{c}^{*} \bar{D}\right)\right], \Gamma_{P_{c}^{+}(4380)}^{3 / 2-}\left[\left(\Sigma_{c}^{*} \bar{D}^{*}\right)\right] \Gamma_{P_{c}^{+}(4450)}^{3 / 2^{-}}\left[\left(\Sigma_{c}^{*} \bar{D}\right)\right]$, and $\Gamma_{P_{c}^{+}(4450)}^{3 / 2^{-}}\left[\left(\Sigma_{c}^{*} \bar{D}^{*}\right)\right]$ are 102.9, 205.9, 67.5, and $135 \mathrm{MeV}$, respectively. The much larger partial decay width $\Gamma_{P_{c}^{+}(4450)}^{3 / 2^{-}}\left[\left(\Sigma_{c}^{*} \bar{D}^{*}\right)\right]$, comparing to the data, excludes the possibility of $P_{c}(4450)$ as a $S$-wave $J^{P}=3 / 2^{-} \Sigma_{c}^{*} \bar{D}^{*}$ molecule. Also, the $P_{c}(4450)$ as the $\Sigma_{c}^{*} \bar{D}$ system is not favored due to its higher mass over the threshold and a slightly large partial decay width. The above discussion shows that if the $P_{c}(4450)$ state has the spin-parity $J^{P}=3 / 2^{-}$, only $\Sigma_{c} \bar{D}^{*}$ system, out of the three molecular scenarios, is allowed.

\section{Summary}

In this paper we show our estimate for the production of the two neutral $P_{c}$ states in the $\pi^{-} p \rightarrow$ $J / \psi n$ process, which may be measured in the future experiment at J-PARC. The calculated differential cross sections are expected to give more information about the spin-parities of the two states. In 
the calculation, the partial branching ratios of $P_{c} J / \psi N$ and $P_{c} \pi N$ are assumed to be $10 \%$ and $1 \%$. Moreover, we also show a calculation for the strong decay of $P_{c} \rightarrow J / \psi p$ based on a $\Sigma_{c} \bar{D}^{*}$ molecule scenario. The estimated widths exclude all the $P$-wave $\Sigma_{c} \bar{D}^{*}$ assumptions in this case. For the $J^{P}=$ $3 / 2^{-} \Sigma_{c}^{*} \bar{D}$ and $\Sigma_{c}^{*} \bar{D}^{*}$ scenarios, only $\Sigma_{c}^{*} \bar{D}$ for $P_{c}(4380)$ case might be possible. Particularly, the $S$ wave $\Sigma_{c} \bar{D}^{*}$ assignments for $P_{c}(4380)$ and $P_{c}(4450)$ may be both favored by the experimental data. It should be noted that this conclusion contradicts to the fact that the two $P_{c}$ states have different spins and opposite parities. Since $P_{c}(4380)$ is broad while $P_{c}(4450)$ is narrow, and $P_{c}(4450)$ is much closer to the threshold of $\Sigma_{c} \bar{D}^{*}$, one should be careful to draw any conclusion for the $P_{c}(4380)$ state. This indicates an agreement with the interpretations of [8], in which the $P_{c}(4450)$ is a $J^{P}=3 / 2^{-} \Sigma_{c} \bar{D}^{*}$ resonance and $P_{c}(4380)$ may be not a genuine state. Theoretical investigations on other decay modes and further experimental information on the spin-parities and the partial decay widths will be helpful to understand the nature of the two $P_{c}$ states.

\section{References}

[1] R. Aaij et al. [LHCb Collaboration], Phys. Rev. Lett. 115, 072001 (2015)

[2] R. Aaij et al. [LHCb Collaboration], Chin. Phys. C 40, 011001 (2016)

[3] R. Aaij et al. [LHCb Collaboration], Phys. Rev. Lett. 117, 082003 (2016)

[4] X. Liu, Chin. Sci. Bull. 59, 3815 (2014)

[5] H. X. Chen, X. Liu, and S. L. Zhu, Phys. Rept. 639, 1-121 (2016)

[6] L. Maiani, A. D. Polosa, and V. Riquer, Phys. Lett. B 749, 289 (2015)

[7] R. F. Lebed, Phys. Lett. B 749, 454 (2015)

[8] M. Karliner and J. L. Rosner, Phys. Rev. Lett. 115, 122001 (2015)

[9] R. Chen, X. Liu, X. Q. Li, and S. L. Zhu, Phys. Rev. Lett. 115, 132002 (2015)

[10] J. He, Phys. Lett. B 753, 547 (2016)

[11] U. -G. Meiner and J. A. Oller, Phys. Lett. B 751, 59 (2015)

[12] M. I. Eides, V. I. Petrov, and M. V. Polyakov, Phys. Rev. D 93, 054039 (2016)

[13] Q. F. Lü and Y. B. Dong, Phys. Rev. D 93, 074020 (2016)

[14] F. K. Guo, U. -G. Meiner, W. Wang, and Q. Zhao, Phys. Rev. D 92, 071502 (2015)

[15] X. H. Liu, Q. Wang, and Q. Zhao, Phys. Lett. B 757, 231 (2016)

[16] G. J. Wang, L. Ma, X. Liu, and S. L. Zhu, Phys. Rev. D 93, 034031 (2016)

[17] Q. Wang, X. H. Liu and Q. Zhao, Phys. Rev. D 92, 034022 (2015)

[18] V. Kubarovsky and M. B. Voloshin, Phys. Rev. D 92, 031502 (2015)

[19] M. Karliner and J. L. Rosner, Phys. Lett. B 752, 329 (2016)

[20] A. N. H. Blin, C. Fernandez-Ramirez, A. Jackura, et al., Phys. Rev. D 94, 034002 (2016)

[21] Q. F. Lü, X. Y. Wang, J. J. Xie, X. R. Chen, and Y. B. Dong, Phys. Rev. D 93, 034009 (2016)

[22] Y. B. Dong, A. Faessler, T. Gutsche, and V. E. Lyubovitskij, Phys. Rev. D 77, 094013 (2008)

[23] Y. B. Dong, A. Faessler, T. Gutsche, et al., Phys. Rev. D 79, 094013 (2009)

[24] Y. B. Dong, A. Faessler, T. Gutsche, and V. E. Lyubovitskij, J. Phys. G 38, 015001 (2011)

[25] S. H. Kim, S. I. Nam, Y. Oh and H. C. Kim, Phys. Rev. D 84, 114023 (2011)

[26] Q. F. Lü, J. J. Xie and D. M. Li, Phys. Rev. C 90, 034002 (2014)

[27] J. J. Wu and T.-S. H. Lee, Phys. Rev. C 88, 015205 (2013)

[28] J. J. Xie, B. S. Zou and H. C. Chiang, Phys. Rev. C 77, 015206 (2008)

[29] S. Weinberg, Phys. Rev. 130, 776 (1963) 